as a subcoronary bioprosthesis. The patient was transferred from another hospital with fever and symptoms of heart failure as a result of prosthetic valve endocarditis and severe aortic regurgitation. TTE showed vegetation $(0.7 \times 0.3 \mathrm{~cm})$ of 1 leaflet, resulting in severe incompetence, and her blood cultures were positive for Staphylococcus warneri. Because of persistence of heart failure, surgery was performed before completion of the antibiotic course. All leaflets were removed, and an Edwards INTUITY Elite 19-mm valve was implanted inside the stentless graft. At 6 months of follow-up, she was in good general health with no sign of residual infection; TTE showed a well-functioning aortic prosthesis, no PVLs, an ejection fraction of $50 \%$, peak pressure gradient of $10 \mathrm{~mm} \mathrm{Hg}$, and MPG of $6 \mathrm{~mm} \mathrm{Hg}$.

\section{DISCUSSION}

Reoperation after stentless AVR is challenging and frequently requires aortic root replacement. The University of Toronto reported an incidence of $63 \%$ for the Bentall procedure in rAVR as a result of severe adhesions between the stentless valve and the native aortic root. ${ }^{3}$ In case 1 , we observed extreme calcification of the entire Freestyle bioprosthesis and native aortic root. After considering the Bentall procedure, we used the RDV, which allowed us to fix the prosthesis quickly and easily in the left ventricular outflow tract by the frame stent with only 3 guiding sutures at the nadir of each sinus (Figure 1).

The 23-mm Elite also showed excellent hemodynamic performance at follow-up, with MPG of $6 \mathrm{~mm} \mathrm{Hg}$ and no PVLs. In case 2 , the infection was limited in one cusp. After resection of the leaflets, the remaining annulus of the bioprosthesis and the native aortic root were completely blended, and the left main coronary was attached to the annulus.

The internal diameter was $19 \mathrm{~mm}$. The RDV system enabled positioning of the prosthesis with no risk of coronary occlusion, because the guiding suture placed in the nadir of the left coronary sinus ensured correct intraannular positioning of the prosthesis.

Although further investigations are needed, this more conservative surgical technique with RDV introduction inside the stentless graft represents a new additional treatment option for patients requiring rAVR, making the operation easier and potentially less dangerous. Furthermore, it is associated with excellent hemodynamic performance.

\section{References}

1. Joint Task Force on the Management of Valvular Heart Disease of the European Society of Cardiology (ESC); European Association for Cardio-Thoracic Surgery (EACTS); Vahanian A, Alfieri O, Andreotti F, Antunes MJ, Baròn-Esquivias G, Baumgartner H, et al. Guidelines on the management of valvular heart disease (version 2012). Eur Heart J. 2012;33:2451-96.

2. Nishimura RA, Otto CM, Bonow RO, Carabello BA, Erwin JP III, Guyton RA, et al ACC/AHA Task Force Members. 2014 AHA/ACC guideline for the management of patients with valvular heart disease: executive summary: a report of the American College of Cardiology/American Heart Association Task Force on Practice Guidelines. Circulation. 2014;129:2440-92. Erratum in: Circulation. 2014;129:e650.

3. Borger MA, Prasongsukarn K, Armstrong S, Feindel CM, David TE. Stentless aortic valve reoperations: a surgical challenge. Ann Thorac Surg. 2007;84: 737-43; discussion 743-4.

4. Finch J, Roussin I, Pepper J. Failing stentless aortic valves: redo aortic root replacement or valve in a valve? Eur J Cardiothorac Surg. 2013;43:495-504.

5. Gariboldi V, Grisoli D, Devin A, Nee L, Theron A, Hubert S, et al. Reoperation for failure of freestyle bioprosthesis using an Edwards Intuity valve. Ann Thorac Surg. 2013;96:e47-8.

\title{
Cryptic recurrent mitral valve excrescences: A rare cause of stroke
}

\author{
David Rose, MD, ${ }^{a}$ Andrew Knowles, MD, ${ }^{a}$ Mark Sissons, MD, ${ }^{b}$ and Joseph Zacharias, FRCS (CTh), \\ Blackpool, United Kingdom
}

\footnotetext{
From the ${ }^{a}$ Lancashire Cardiac Centre, Blackpool, United Kingdom; and the ${ }^{\mathrm{b}}$ Department of Pathology, Blackpool Victoria Hospital, Blackpool, United Kingdom.

Disclosures: J.Z. is a proctor for Edwards Lifesciences and is reimbursed for the time spent proctoring. All other authors have nothing to disclose with regard to commercial support.

Received for publication Jan 24, 2015; revisions received March 1, 2015; accepted for publication March 26, 2015; available ahead of print April 22, 2015.

Address for reprints: David Rose, MD, Lancashire Cardiac Centre, Whinney Heys Rd, Blackpool, Lancashire FY3 8NR, United Kingdom (E-mail: davidrose@ libero.it).

J Thorac Cardiovasc Surg 2015;149:e89-91

$0022-5223 / \$ 36.00$

Copyright (c) 2015 by The American Association for Thoracic Surgery

http://dx.doi.org/10.1016/j.jtcvs.2015.03.043
}

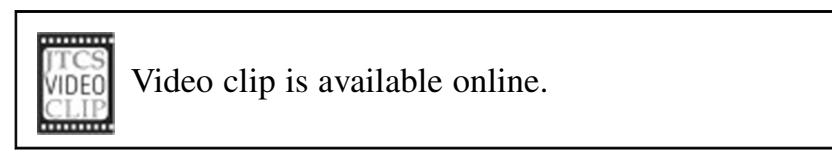

Mesothelial monocytic intracardiac excrescence is considered a benign cardiovascular lesion, but because of its very particular morphology and considering its recurrent behavior, we suggest for this rare valve lesion a strategy to replace the valve rather than attempting a repair. 


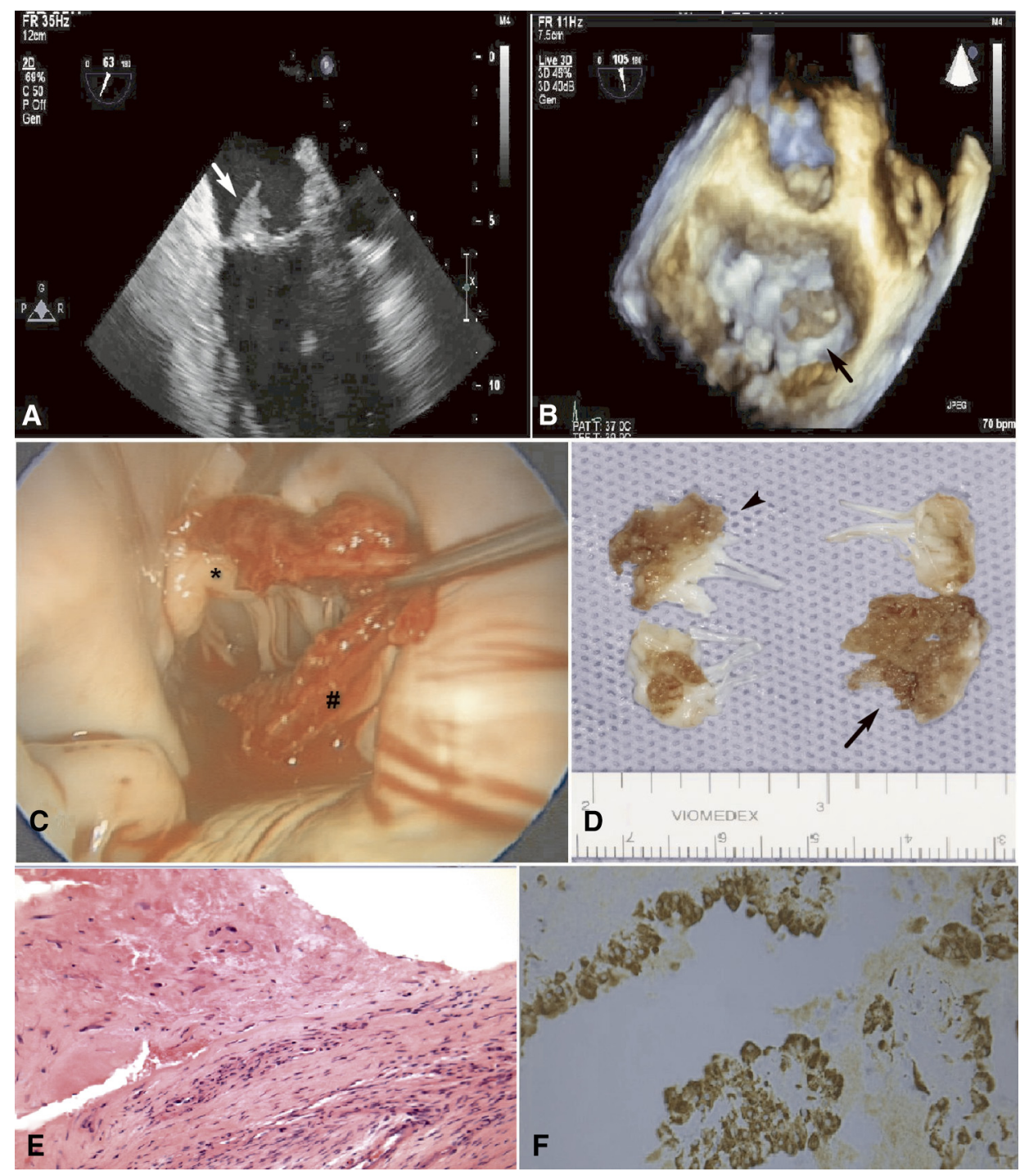

FIGURE 1. A, A transesophageal echocardiographic study clearly showing a mass attached to the base of the anterior mitral valve leaflet (white arrow) and measuring around $1.0 \times 1.7 \mathrm{~cm}$. B, Three-dimensional reconstruction of the mitral valve in which the mass is visible (black arrow). C, Intraoperative aspect of the lesion, with the micromultinodular aspect on the atrial surface of the lesion (asterisk indicates the anterior leaflet; hash mark indicates the posterior leaflet). D, Gross inspection of the valve, showing the lesion on both leaflets (posterior leaflet on the left, indicated by the tip of black arrowhead; anterior leaflet on the right, indicated by black arrow). E, Histologic evaluation showing a mixture of mesothelial cells and fibrin, devoid of elastic fibers, among which are spindle-shaped mesothelial cells, as identified by their positivity with calretinin and pankeratin antibodies (hematoxylin and eosin stain). F, The histiocytes are diffusely immunoreactive for CD68 and MAC387, confirming their macrophage, monocytic nature (diaminobenzidine stain).

\section{CLINICAL SUMMARY}

A 48-year-old man was admitted to the emergency department with recent onset of left hemianopia and dysarthria. A brain magnetic resonance imaging scan revealed a right occipital infarct. An echocardiogram was performed to find the source of his stroke, and it revealed a mass on the anterior leaflet of the mitral valve. Transesophageal echocardiography was performed to define the mass better; this showed a mobile mass attached to the base of the anterior mitral valve leaflet that measured approximately $1.7 \times 1.0 \mathrm{~cm}$ and was interpreted as a possible papillary fibroelastoma (Figure 1, $A$ and $B$, and Video 1 ). The patient had no history of malignancy. Factor V Leiden was not detected. There was no mitral regurgitation. Chest radiography and electrocardiography at admission were unremarkable. Computed tomographic cardiac angiography was then performed before the scheduled operation for the removal of the mass, and apart from normal coronary arteries it confirmed the presence of a hypoattenuating, mobile, frondlike lesion arising from the posterior commissure, $\mathrm{A} 3$ and $\mathrm{P} 3$ of the mitral valve. Blood cultures were sterile. 
Tests for the human immunodeficiency virus, cytomegalovirus, Epstein Barr virus, Q fever, and Chlamydia, Mycoplasma, Bartonella, and Brucella species yielded negative results, as did tests for rheumatoid factor and anticardiolipin antibody (IgG and $\operatorname{IgM}$ ). The patient underwent a minimally invasive approach to mitral valve exploration, and intraoperatively the mass was found to involve the leaflets at the site of the posterior commissure, with the scallops of A3 and P3 completely replaced by the lesion. ${ }^{1,2}$

Macroscopically, the lesion did not share the characteristics of a fibroelastoma. In fact, the leaflet was covered with many caviarlike granules on the atrial surface of the valve (Figure 1, $C$, and Video 2). After the excision of $\mathrm{A} 3$ and $\mathrm{P} 3$ (Figure $1, D$ ), the mitral valve was repaired with an edge-to-edge running suture, where a 4-0 Prolene suture (Ethicon Inc, Somerville, NJ) was used to close of the defect, ensuring that there was a reasonable area of valve opening between $\mathrm{A} 1, \mathrm{~A} 2$, and $\mathrm{P} 1$ and $\mathrm{P} 2{ }^{3}$ The excised tissue was sent for histopathologic, culture, and polymerase chain reaction (PCR) studies.

Because of its very peculiar architecture, it is easy to understand the uncommon embolic behavior of this type of lesion.

Cultures failed to show any growth of microorganisms, and the PCR did not show any bacterial DNA. Histologic examination showed the lesions to be covered by plump mononuclear cells, which were immunohistochemically positive with antibodies to CD68 and MAC387, confirming their macrophage, monocytic nature. Deep to this was abundant fibrin, devoid of elastic fibers, among which were spindle-shaped mesothelial cells, as identified by their positivity with calretinin and pankeratin antibodies ${ }^{4}$ (Figure 1, $E$ and $F$ ).

The first follow-up at 3 months was normal. The patient was free of symptoms, and echocardiography showed the repaired mitral valve working well, with a mean gradient of $6 \mathrm{~mm} \mathrm{Hg}$ (Video 3).

Unfortunately, the patient was readmitted to our unit 8 months after surgery with increased shortness of breath. The transesophageal echocardiographic study (Video 4) showed mitral valve with a thickened leaflet, an area of 7 $\mathrm{cm}^{2}$, a mean gradient of $17 \mathrm{~mm} \mathrm{Hg}$, and a peak gradient of $27 \mathrm{~mm} \mathrm{Hg}$.

We repeated a computed tomographic angiographic pulmonary scan to rule out any pulmonary embolism. The patient underwent an elective sternotomy for a mitral valve reexploration, and intraoperatively the mass was very similar to the previous mass and involved both leaflets at the site of commissures and the implanted ring (Video 5). We then proceeded to excise the valve in its entirety, and after resuspending the papillary muscles we inserted a mechanical mitral valve in a standard fashion. The excised tissue was sent for histopathologic, culture, and PCR studies. As previously, on this occasion cultures failed to show any growth of microorganism and PCR results were negative. Histologic examination showed marked vascular proliferation and numerous plump endothelial cells (positive with antibodies Q-BEnd). The superficial part of the valve cusp showed prominent myxoid degeneration with a large amount of fibrin.

At the 12-month follow-up, the patient was completely free of symptoms, with the mechanical valve working well and with no echocardiographic signs of recurrence.

\section{DISCUSSION}

The diagnosis was mesothelial monocytic intracardiac excrescence. Mesothelial monocytic intracardiac excrescence, which is considered a benign cardiovascular lesion, is composed of a mixture of histiocytes, mesothelial cells, fibrin, adipocytes, and scattered inflammatory cells without a vascular network or supporting stroma. ${ }^{4}$

There are 2 theories explaining the cause of this rare lesion. The reactive theory attributes a pathogenic role to mechanical irritation, inflammation, or neoplasm as possible triggers for the formation of the lesion. The artifactual hypothesis supports an iatrogenic clustering of free-floating cells that enters the heart as the result of cardiac surgery.

Mesothelial monocytic intracardiac excrescence is considered a benign lesion. Because of its very particular morphology and considering its recurrent behavior, however, we suggest a strategy to replace the valve rather than attempting a repair for this rare valve lesion.

\section{References}

1. Chirichilli I, D’Ascoli R, Rose D, Frati G, Greco E. Port Access (Thru-Port System) video-assisted mitral valve surgery. J Thorac Dis. 2013;5(Suppl 6): S680-5.

2. Cohn LH, Byrne JG. Minimally invasive mitral valve surgery: current status. Tex Heart Inst J. 2013;40:575-6.

3. Gaur P, Kaneko T, McGurk S, Rawn JD, Maloney A, Cohn LH. Mitral valve repair versus replacement in the elderly: short-term and long-term outcomes. J Thorac Cardiovasc Surg. 2014;148:1400-6.

4. Hu ZL, Lü H, Yin HL, Wen JF, Jin O. A case of mesothelial/monocytic incidental cardiac excrescence and literature review. Diagn Pathol. 2010;5:40.

5. Courtice RW, Stinson WA, Walley VM. Tissue fragments recovered at cardiac surgery masquerading as tumoral proliferations. Evidence suggesting iatrogenic or artefactual origin and common occurrence. Am J Surg Pathol. 1994;18: 167-74. 